\title{
Breeding Temperate Fruit Crops for Improved Freezing Tolerance
}

\author{
Christopher L. Owens \\ U.S. Department of Agriculture, Agricultural Research Service, Plant Genetic Resources Unit, Cornell University, \\ Geneva, NY 14456
}

\begin{abstract}
Low temperature is one of the most important environmental factors limiting crop plant growth, distribution, and productivity. New cultivars with improved freezing tolerance are a common breeding objective of many temperate fruit breeding programs. Improved freezing tolerance would prevent crop loss due to low temperature and reduce yearly fluctuations in crop quantity and quality. Breeding temperate fruit cultivars for improved freezing tolerance is made difficult by several factors, including complexity of the phenotype, difficulty in accurate measurement of the phenotype, and lack of fundamental knowledge concerning the inheritance and genetic control of this trait. Results from inheritance studies of freezing tolerance in temperate fruit crops as well as recent research in forestry genetics highlight some of the challenges and opportunities for further elucidating the inheritance of freezing tolerance in temperate fruit crops. A tremendous amount of research has been conducted describing the molecular biology and signal transduction of the cold acclimation response in the model plant, Arabidopsis thaliana. These findings have begun the transfer to research in agriculturally important crops and hold great promise for elucidating novel methods for generating new fruit cultivars with improved freezing tolerance.
\end{abstract}

The generation of new fruit cultivars with improved freezing tolerance is a challenging prospect, and is considered by some to be an intractable trait. What are the aspects of freezing tolerance that make the breeding of this trait intractable? The first difficulty is the complexity of the phenotype. The breeder needs to tailor the breeding objectives to the improvement of freezing tolerance and plant part that makes most sense to the breeder in their particular production regions. For example, is midwinter damage to stems or buds most likely, or deacclimation of flowerbuds due to fluctuating spring temperatures and subsequent late frosts? Additionally, freezing tolerance is not a static parameter and can vary over time, developmental stage, day length, historical cropload, ambienttemperature, etc. The enormous complexity of the freezing tolerance phenotype places the burden on the breeder and geneticist to exercise as much precision as is feasible in the screening for freezing tolerance and the description of phenotype. Methodology used for the ascertainment of freezing tolerance is anothermajor source of complexity. Decisions on controlled or natural environment screening, and choice of methods for assaying freezing damage create numerous challenging decisions for the breeder. Lack of variation in the trait of interest can be a difficulty contributing to the intractability of this trait. Is germplasm available for the trait of interest? How much variation exists within the gene pool of a particular fruit crop for improving freezing tolerance. In addition, we havevery little data on the inheritance of freezing tolerance in any temperate fruit crop. Studies to determine the heritability, gene action, or genomic location of quantitative trait loci are limited at best in the temperate fruit crops genetics literature. Recent research in other nonfruit-bearing woody plants highlights some of the challenges and opportunities for further elucidating the inheritance of freezing tolerance in temperate fruit crops.

Similarly, a tremendous amount of research has been conducted in recent years describing the molecular biology and signal transduction of the cold acclimation response, primarily in the model plant, Arabidopsis thaliana. These findings in model systems have begun the transfer to additional research in agriculturally important crops, such as perennial fruit crops (Wisniewski et al., 2003). Despite the enormous complexity of the phenotype, the genetic regulation and control of freezing tolerance, and the environmental interactions imposed on the genotype, progress is being made in our understanding of a plant's response to low temperatures. The future is bright for the development of new strategies for the generation of new fruit cultivars with improved freezing tolerance.

\section{Availability of Cold-hardy Germplasm}

Available germplasm resources are a key component to further improvement in fruit cultivars with enhanced freezing tolerance. Numerous wild species have been used as sources of cold hardiness in the breeding of new fruit cultivars (Quamme and Stushnoff, 1983) and for many crops there is ample genetic material available for improving freezing tolerance (Quamme, 1978). In many cases the challenge is not identifying sources of cold hardiness, but the simultaneous selection for multiple quantitative traits in breeding populations, such as cold hardiness, fruit quality, disease resistance, productivity, plant architecture, etc. Of course, the availability of cold hardy germplasm differs amongst species, and the improvement of some traits/crops is limited by the germplasm available. For example, significant progress has been made in generating hardy wine grape cultivars through the hybridization of Vitis riparia and either $V$. vinifera or complex Vitis hybrids (Hemstad and Luby, 2000). In contrast the extent of possible improvement of the hardiness of many cultivated Prunus species appears to be more limited (Quamme, 1978). In this regard, continued collection, characterization, and distribution of wild germplasm are an essential aspect in the future success of generating fruit cultivars with improved freezing tolerance.
The Inheritance of Freezing Tolerance in Temperate Fruit Crops

Detailed studies on the inheritance of aspects of freezing tolerance are few and far between within the fruit crop genetics literature (Quamme 1978; Stushnoff 1972). The same conditions that make the breeding of cold hardy fruit cultivars challenging or intractable present challenges in conducting experiments to elucidate the genetic mechanisms of freezing tolerance. Areview of the literature on this topic (Table 1) shows that heritability estimates have been generated for several aspects of freezing tolerance in a few fruit crops, and generally the values that are reported are moderate to high (Arora et al., 1992; Ashworth and Wisniewski, 1991; Howe etal., 2003; Luby, 1991; Quamme, 1978). Genetic components of variance have not frequently been obtained for freezing tolerance in fruit crops, but additive variance has been reported as of major importance in apple and blueberry (Arora et al., 2000; Fear et al., 1985; Watkins and Spangelo, 1970). Generally, all that is known concerning the inheritance of freezing tolerance in many fruit crops is that the trait is quantitatively inherited and that cold hardiness appears transferable to progeny. The moderate to high levels of heritability reported in the literature again suggest that identifying cold hardy germplasm and transferring that trait to future generations is not difficult, but rather the combination of multiple quantitative traits, chiefly cold hardiness and fruit quality is the challenge.

More detailed quantitative genetic analyses using molecular markers and genetic linkage maps to identify and localize loci of importance within the genome have been conducted in several forestry species in recent years that can help inform fruit crop breeders and geneticists (reviewed in Howe et al. (2003).

Table 1. Heritability of freezing tolerance in selected temperate fruit crops.

\begin{tabular}{llll}
\hline Crop & Phenotypic trait & Heritability & Citation \\
\hline Plum & Winter survival & $>0.0$ & Dorsey and Bushnell, 1925 \\
Peach & Cambium, xylem, and bud injury & $0.65-0.96$ & Cain and Anderson, 1980 \\
Peach & Dormant flower buds & Mid-high & Mowry, 1964 \\
Blueberry & Bud freezing tolerance & High & Arora et al., 2000; Fear et al., 1985 \\
Apple & Winter survival & Mid-high & Fejer, 1976; Watkins and Spangelo, 1970 \\
\hline
\end{tabular}


These reports show the heritability of numerous aspects of freezing tolerance to be quite low. For example, in douglas fir spring frost damage was observed to have a heritability of 0.56 and the heritability of midwinter hardiness was 0.11 (Jermstad et al., 2001a, 2001b, 2003). Several quantitative trait loci (QTL) analyses have been conducted on forestry species and these experiments have generally shown that QTLs for freezing tolerance can be identified and localized within a genome, but that the percentage of phenotypic variation explained by an individual QTL are generally low, but is some cases can explain $>25 \%$ of the phenotypic variation (Chen et al., 2002; Jermstad et al., 2001a, 2001b). These heritability values are considerably lower than the few reported for most fruit crops. The discrepancy could be due to experimental methodology, or nature of genetic variation within the populations under study. Additionally, recent work describing an analysis for freezing tolerance in citrus has shown results similar to those present for spruce, poplar, and douglas fir (Weber et al., 2003). To my knowledge, results from QTL analysis of freezing tolerance in a temperate fruit crop have not yet been reported in the literature. Better knowledge of the quantitative genetics of freezing tolerance in temperate fruit crops should aid breeders in their efforts. In particular, information on location of specific QTL, molecular markers tightly linked to those QTL, and an estimation of phenotypic importance of those loci should improve the efficiency of efforts to breed new cultivars with enhanced freezing tolerance.

\section{Lessons from Arabidopsis}

In recent years tremendous advances have been made in our understanding of the molecular regulation of cold acclimation in the model plant Arabidopsis thaliana. Cold acclimation is the process by which a plant exposed to low, but nonlethal temperature subsequently develops improved freezing tolerance. These studies are leading to increasingly complex descriptions of the signal transduction pathways leading from the plant's, or a plant cell's, perception of a cold stimulus to the molecular triggers of the cold acclimation process and the eventual attainment of increased freezing tolerance. Transfer of this knowledge gained from studies of arabidopsis to the improvement of horticultural crops including temperate fruit species is an ongoing challenge. While questions exist concerning the utility of arabidopsis as a model for understanding cold acclimation in a perennial fruit crop, there is no question that there is tremendous genetic conservation between individual genes and signal transduction pathways identified in arabidopsis and numerous agriculturally important plants, including fruit crops.

Many low-temperature induced genes have been identified in several temperate fruit species (Arora and Wisniewski, 1994; Cai et al., 1995; Dhanaraj et al., 2004; Kitashiba et al., 2002; Muthalif and Rowland, 1994; Owens et al., 2002; Porat et al., 2002; Tian et al., 2002). These genes have been identified both through observations of differential gene and protein expression in the crops of interest and also by searching for orthologous sequences of previously identified genes first characterized in arabidopsis and other model plant species. The challenge for the fruit breeder or geneticist is to identify specific genes that can be targets for selection in breeding programs or for biotechnological manipulation. Many low-temperature induced genes that have been characterized in model species are now being examined in fruit crop species. Much of this work seeks to discover candidates suitable for creating transgenic crops with enhanced levels of freezing tolerance (Sung et al., 2003; Zhang et al., 2004). In addition, research elucidating the underlying natural genetic variation in freezing tolerance within our important crop plants through combining quantitative genetics with characterization of variation within candidate genes of interest is increasing and may lead to novel approaches to understanding the inheritance of freezing tolerance is agriculturally important plants.

\section{Identification of Candidate Freezing Tolerance Genes}

Many genes have been identified that are regulated in response to low temperature in plants. Our knowledge of how these genes are regulated and their exact role in freezing tolerance is still incomplete. Two main approaches have been taken to better understand the factors that regulate this large number of cold-induced genes. One approach, based on the differential expression of either proteins or mRNA transcripts has either directly or indirectly lead to the identification of regulatory proteins involved in the regulation of cold-induced gene expression (Gilmour et al., 2000; Kim et al., 2001; Stockinger et al., 1997). A second approach has been to identify mutants with altered cold acclimation or freezing tolerance phenotypes. In Arabidopsis, this mutational approach has identified numerous genes within the cold acclimation signal transduction pathways such as the sensitivity to freezing (sfr) mutants that do not cold acclimate (Warren et al., 1996), eskimol that shows constitutive freezing tolerance even at warm temperatures (Xin and Browse, 1998), and HOS1, which was identified by possessing heightened expression of several cold-regulated genes following cold treatment (Ishitani et al., 1998). New genetic lesions that cause alterations in the cold acclimation signal transduction pathways in arabidopsis continue to be identified, their positions placed within the signal cascades, and characterized at a rapid pace (Chinnusamy et al., 2004). The combined data from both of these approaches is beginning to provide a framework for understanding plant gene expression in response to cold. Cold signaling pathways and networks appear to be complex, and have many points in parallel as well as many interactions (Ishitani et al., 1997). As these pathways are further defined, opportunities exist to identify key regulatory proteins or branch-points that may be suitable for targets to improve the freezing tolerance of temperate fruit crops.

\section{CBF-COR Genes}

Perhaps the best characterized cold-induced genes are the arabidopsis cor genes. In Arabidopsis, studies of the molecular basis of cold acclimation have identified a common cis-acting regulatory element, the C-repeat/Dehydration Responsive Element (CRT/DRE), which is defined as a CCGAC, within the promoters of several cold induced $(C O R)$ genes (Stockinger et al. 1997). In turn, all of the currently characterized arabidopsis $C O R$ genes are coordinately up-regulated by $C B F 1, C B F 2$, or $C B F 3$, a family of cold and drought inducible transcriptional activators that bind to promoters containing a CRT/DRE (Haake et al., 2002; Jaglo-Ottosen et al., 1998; Kasuga et al., 1999; Liu et al., 1998; Medina et al., 1999; Thomashow, 2001). Even though freezing tolerance is a complexly inherited trait, manipulation of just one gene, $C B F 1$, has been shown to improve the whole plant freezing tolerance of arabidopsis and Brassica napus (Jaglo et al., 2001; Jaglo-Ottosen et al., 1998). Additionally, it is known that these COR genes can be up-regulated by additional transcription factors DREB2A and DREB2B, which are important in the response to dehydration (Liu et al., 1998).

CBFs have been identified in many crop species including tomato, barley, Brassica, and Prunus (Choi et al., 2002; Dubouzet et al., 2003; Jaglo et al., 2001; Owens et al., 2002). In these crops, these $\mathrm{CBF}$-like proteins are cold-regulated in a fashion similar to CBF1, 2, and 3 of arabidopsis, and possess high levels of amino acid identity. Additionally, overexpression of $C B F 1,2$, or 3 in Brassica napus increases its freezing tolerance and up-regulates Bn115 and Bn28, homologs of COR15a and COR 6.6 of arabidopsis. These findings suggest that the CBF-COR system is highly conserved in diverse plant families, and may be a key regulator of cold temperature stress tolerance in many plants. As mentioned above, the CBF family of transcriptional activators are important regulators of cold acclimation in arabidopsis. When overexpressed these genes have been shown to enhance the freezing tolerance of both arabidopsis and B. napus, and orthologs of the $C B F$ gene family have been identified in a diverse array of plant species, such as wheat, rye, and tomato (Jaglo et al., 2001; Kitashiba et al., 2004; Owens et al., 2002). Considering that the $C B F$ transcriptional regulators appear to be widely conserved within cultivated plants and that over-expression of CBFs has shown the capability of enhancing freezing tolerance in several crop species (Jaglo et al., 2001; Kasuga et al., 1999; Kitashiba et al., 2004; Owens et al., 2002) there has been considerably interest in further characterizing these genes in crop plants.

\section{Integrating Gene Expression to Physiological Responses to Cold}

Many physiological and biochemical changes are known to occur in plants in response to low temperature. These changes include alterations in lipid metabolism, changes 
in the properties of membranes (Murata and Los, 1997), shifts in carbohydrate production and localization, accumulation of proline, betaine, and other compatible osmolytes (Levitt, 1980), and shifts in plant secondary metabolism (Guy, 1990). However, very few of the cold-induced genes characterized in plants have been directly tied to a known physiological function. Linking the large number of cold-induced physiological and biochemical changes to the molecular genetics of cold acclimation is a major future challenge.

Cold induced genes fall into many categories including lipid desaturases, antifreeze proteins, and superoxide dismutases; the largest number having unknown functions. A large subset of the genes of unknown function share similarity with a class of proteins known as LEA proteins, which were first described in cereals and are transcribed late in embryogenesis during seed desiccation (Thomashow, 1999). Even though some of these cold-induced genes share homology with LEA proteins and some do not, many of them share several common characteristics. Common traits include proteins that are highly hydrophilic, possess simple amino acid compositions, remain soluble upon boiling in aqueous buffer, and have many simple repeated amino acid motifs (Thomashow, 1999). More detailed studies of one of these genes, cor15a from Arabidopsis, suggest that the COR15am protein has the ability to shift downward the temperature at which the lamellar to hexagonal II phase transition occurs in plant membranes (Steponkus et al., 1998). This phase transition is known to lead to extensive cellular damage upon return of the cells to non-freezing temperatures. This temperature shift allows the cells to withstand lower freezing temperatures without suffering damage. In the case of COR15am, the inner membrane of the chloroplast is thought to be the binding target, and it is hypothesized that this protein has the ability to alter the intrinsic curvature of the membrane leading to the temperature shift of the phase transition (Steponkus et al., 1998). Since many of the cold-induced genes share properties with COR15a it is possible that membrane stability is a common mode of freezing tolerance in plants. However, little is known about the physiological role of most cold-induced plant genes.

\section{Study of Naturally Occurring Variation in Freezing Tolerance}

Few detailed studies on the genetic variation of freezing tolerance in temperate fruit species have been reported. QTL analysis of freezing tolerance have been conducted for numerous plant species including citrus and several forestry species. These studies typically reinforce findings from classical quantitative genetics, primarily that freezing tolerance is quantitatively inherited, there are numerous important loci in many regions of the genome and that occasionally there appears to be some loci that explain larger proportions of the phenotypic variation within a given population. Initial reports assessing the co-localization of candidate genes for freezing tolerance with mapped QTL for freezing tolerance have been reported in poplar and wheat (Chen et al., 2002; Vagujfalvi et al., 2003). These findings suggest that characterization of candidate genes identified first in model species in crop plants of interest has potential not only for transgenic manipulation of freezing tolerance but may help explain fundamental questions concerning genotypic variation in freezing tolerance within cultivated plants and their wild relatives. As increasing numbers of freezing tolerance candidate genes are identified in fruit species, there will be more opportunities to combine quantitative genetics with molecular analysis to identify important loci underlying the cold acclimation response.

These findings in model systems have begun the transfer to additional research in agriculturally important crops, such as perennial fruit crops. Despite the enormous complexity of the phenotype, the genetic regulation and control of freezing tolerance, and the environmental interactions imposed on the genotype, progress is being made in our understanding of a plant's response to low temperatures. Much of the recent progress in our understanding of the genetics of freezing tolerance in temperate fruit crops is predicated on research conducted in Arabidopsis. As this transfer of knowledge continues and as novel genes are identified within crop plants of interest, there will be increasing opportunities for targets to aid in the generation of new fruit cultivars with enhanced freezing tolerance.

\section{Literature Cited}

Arora, R., L.J. Rowland, J.S. Lehman, C.C. Lim, G.R. Panta, and N. Vorsa. 2000. Genetic analysis of freezing tolerance in blueberry (Vaccinium section Cyanococcus). Theor. Appl. Genet. 100:690-696.

Arora, R. and M. Wisniewski. 1994. Cold acclimation in genetically related (Sibling) deciduous and evergreen peach (Prunus persica [L.] Batsch) II. A 60-kilodalton bark protein in cold-acclimated tissues of peach is heat stable and related to the dehydrin family of proteins. Plant Physiol. 105:95-101.

Arora, R., M.E. Wisniewski, and R. Scorza. 1992. Cold acclimation in genetically related (Sibling) deciduous and evergreen peach (Prunus persica [L.] Batsch). Plant Physiol. 99:1562-1568.

Ashworth, E.N. and M.E. Wisniewski. 1991. Response of fruit tree tissues to freezing temperatures. HortScience 26:501-504.

Cain, D.W. and R.L. Anderson. 1980. Inheritance of wood hardiness among hybrids of commercial and wild asian peach genotypes. J. Amer. Soc. Hort. Sci. 105:349-354.

Chen, T.H.H., G.T. Howe, and H.D. Bradshaw. 2002. Molecular genetic analysis of dormancy-related traits in poplars. Weed Sci. 50:232-240.

Chinnusamy, V., K. Schumaker, and J.-K. Zhu. 2004. Molecular genetic perspectives on cross-talk and specificity in abiotic stress signaling in plants. J. Expt. Bot. 55:225-236.

Choi, D.W., E.M. Rodriguez, and T.J. Close. 2002. Barley $C b f 3$ gene identification, expression pattern, and map location. Plant Physiol. 129:1781-1787.

Dorsey, M.J. and J. Bushnell. 1925. Plum Investigations II. The inheritance of hardiness. Univ. Minn. Agr. Expt. Stat. Tech. Bul. 32.

Dubouzet, J.G., Y. Sakuma, Y. Ito, M. Kasuga, E.G. Dubouzet, S. Miura, M. Seki, K. Shinozaki, and
K. Yamaguchi-Shinozaki. 2003. OsDREB genes in rice, Oryza sativa L., encode transcription activators that function in drought-, high-saltand cold-responsive gene expression. Plant $\mathrm{J}$. 33:751-763.

Fear, C.D., F.I. Lauer, J.J. Luby, and R.L. Stucker. 1985. Genetic components of variance for winter injury, fall growth cessation, and off-season flowering in blueberry progenies. J. Amer. Soc. Hort. Sci. 110:262-266.

Fejer, S.O. 1976. Combining ability and correlations of winter survival, electrical impedance and morphology in juvenile apple trees. Can. J. Plant Sci. 56:303-309.

Gilmour, S.J., A.M. Sebolt, M.P. Salazar, J.D. Everard, and M.F. Thomashow. 2000. Overexpression of the arabidopsis $C B F 3$ transcriptional activator mimics multiple biochemical changes associated with cold acclimation. Plant Physiol. 124:1854-1865.

Guy, C.L. 1990. Cold acclimation and freezing stress tolerance: Role of protein metabolism. Annu. Rev. Plant Physiol. Plant Mol. Biol. 41:187-223.

Haake, V., D. Cook, J.L. Riechmann, O. Pineda, M.F. Thomashow, and J.Z.Zhang. 2002. Transcription factor $C B F 4$ is a regulator of drought adaptation in arabidopsis. Plant Physiol. 130:639-648.

Hemstad, P.R. and J.J. Luby. 2000. Utilization of Vitis riparia for the development of new wine varieties with resistance to disease and extreme cold. Acta Hort. 528:487-490.

Howe, G.T., S.N. Aitken, D.B. Neale, K.D. Jermstad, N.C. Wheeler, and T.H.H. Chen. 2003. From genotype to phenotype: Unraveling the complexities of cold adaptation in forest trees. Can. J. Bot. 81:1247-1266.

Ishitani, M., L. Xiong, H. Lee, B. Stevenson, and J.K. Zhu. 1998. HOS1, a genetic locus involved in cold-responsive gene expression in arabidopsis. Plant Cell 10:1151-1161.

Jaglo, K.R., S. Kleff, K.L. Amundsen, X. Zhang, V. Haake, J.Z. Zhang, T. Deits, and M.F. Thomashow. 2001. Components of the arabidopsis Crepeat/dehydration-responsive element binding factor cold-response pathway are conserved in Brassica napus and other plant species. Plant Physiol. 127:910-917.

Jaglo-Ottosen, K.R., S.J. Gilmour, D.G. Zarka, O. Schabenberger, and M.F. Thomashow. 1998. Arabidopsis $C B F 1$ overexpression induces $C O R$ genes and enhances freezing tolerance. Science 280:104-106.

Jermstad, K.D., D.L. Bassoni, K.S. Jech, G.A. Ritchie, N.C. Wheeler, and D.B. Neale. 2003. Mapping of quantitative trait loci controlling adaptive traits in coastal douglas-fir. III. Quantitative trait loci-by-environment interactions. Genetics 165:1489-1506.

Jermstad, K.D., D.L. Bassoni, K.S. Jech, N.C. Wheeler, and D.B. Neale. 2001a. Mapping of quantitative trait loci controlling adaptive traits in coastal douglas-fir. I. Timing of vegetative bud flush. Theor. Appl. Genet. 102:1142-1151.

Jermstad, K.D., D.L. Bassoni, N.C. Wheeler, T.S. Anekonda, S.N. Aitken, W.T. Adams, and D.B. Neale. 2001b. Mapping of quantitative trait loci controlling adaptive traits in coastal douglas-fir. II. Spring and fall cold-hardiness. Theor. Appl. Genet. 102:1152-1158.

Kasuga, M., Q. Liu, S. Miura, K. Yamaguchi-Shinozaki, and K. Shinozaki. 1999. Improving plant drought, salt, and freezing tolerance by gene transfer of a single stress-inducible transcription factor. Nat. Biotech. 17:287-291.

Kim, J.C., S.H. Lee, Y.H. Cheong, C.-M. Yoo, S.I. Lee, H.J. Chun, D.-J. Yun, J.C. Hong, S.Y. Lee, C.O. Lim, and M.J. Cho. 2001. A novel coldinducible zinc finger protein from soybean, 
SCOF-1, enhances cold tolerance in transgenic plants. Plant J. 25:247-259.

Kitashiba, H., T. Ishizaka, K. Isuzugawa, K. Nishimura, and T. Suzuki. 2004. Expression of a sweet cherry DREB1/CBF ortholog in Arabidopsis confers salt and freezing tolerance. J. Plant Physiol. 161:1171-1176.

Liu, Q., M. Kasuga, Y. Sakuma, H. Abe, S. Miura, K. Yamaguchi-Shinozaki, and K. Shinozaki. 1998. Two transcription factors, DREB1 and DREB2, with an EREBP/AP2 DNA binding domain separate two cellular signal transduction pathways in drought- and low-temperature-responsive gene expression, respectively, in arabidopsis. Plant Cell 10:1391-1406.

Luby, J.J. 1991. Breeding cold-hardy fruit crops in Minnesota. Hortscience 26:507-512.

Medina, J., M. Bargues, J. Terol, M. Perez-Alonso, and J. Salinas. 1999. The arabidopsis $C B F$ gene family is composed of three genes encoding AP2 domain-containing proteins whose expression is regulated by low temperature but not by abscisic acid or dehydration. Plant Physiol. 119:463-469.

Mowry, J.B. 1964. Inheritance of cold hardiness of dormant peach flowers. Proc. Amer. Soc. Hort. Sci. 85:128-133.

Murata, N. and D.A. Los. 1997. Membrane fluidity and temperature perception. Plant Physiol. 115:875-879.

NDong, C., F. Ouellet, M. Houde, and F. Sarhan. 1997. Gene expression during cold acclimation in strawberry. Plant Cell Physiol. 38:863-870.

Owens, C.L., M.F. Thomashow, J.F. Hancock, and
A.F. Iezzoni. 2002. $C B F 1$ orthologs in sour cherry and strawberry and the heterologous expression of $C B F 1$ in strawberry. J. Amer. Soc. Hort. Sci. 127:489-494.

Quamme, H.A. 1978. Breeding and selecting temperate fruit crops for cold hardiness. p. 313-332. In: P. H. Li and A. Sakai, (eds). Plant cold hardiness and freezing stress. Academic Press, New York.

Quamme, H.A. and C. Stushnoff. 1983. Resistance to environmental stress, p. 242-266. In: J.N. Moore and J. Janick (eds.). Methods in fruit breeding. Purdue Univ. Press, West Lafayette, Ind.

Steponkus, P.L., M. Uemura, R.A. Joseph, S. J. Gilmour, and M. F. Thomashow. 1998. Mode of action of the COR15a gene on the freezing tolerance of Arabidopsis thaliana. Proc. Nat. Acad. Sci. (USA) 95:14570-14575.

Stockinger, E.J., S J. Gilmour, and M.F. Thomashow. 1997. Arabidopsis thaliana CBF1 encodes an AP2 domain-containing transcriptional activator that binds to the C-repeat/DRE, a cis-acting regulatory element that stimulates transcription in response to low temperature and water deficit. Proc. Nat. Acad. Sci. (USA) 94:1035-1040.

Stushnoff, C. 1972. Breeding for cold hardiness. HortScience 7:10-13.

Sung, D.-Y., F. Kaplan, K.-J. Lee, and C.L. Guy. 2003. Acquired tolerance to temperature extremes. Trends Plant Sci. 8:179-187.

Thomashow, M.F. 1999. Plant cold acclimation: Freezing tolerance genes and regulatory mechanisms. Annu. Rev. Plant Physiol. Plant Mol. Biol. 50:571-599.
Thomashow, M.F. 2001. So what's new in the field of plant cold acclimation? Lots! Plant Physiol. 125:89-93.

Vagujfalvi, A., G. Galiba, L. Cattivelli, and J. Dubcovsky. 2003. The cold-regulated transcriptional activator $C b f 3$ is linked to the frost-tolerance locus $\mathrm{Fr}$ - $\mathrm{A2}$ on wheat chromosome 5A. Mol. Genet. Genom. 269:60-67.

Warren, G., R. McKown, A. Marin, and R. Teutonico. 1996. Isolation of mutations affecting the development of freezing tolerance in Arabidopsis thaliana (L.) Heynh. Plant Physiol. 111:1011-1019.

Watkins, R. and L.P. S. Spangelo. 1970. Components of genetic variance for plant survival and vigor in apple trees. Theor. Appl. Genet. 40:195-203.

Weber, C.A., G. Moore, Z. Deng, and F.G. Gmitter, Jr. 2003. Mapping freeze tolerance quantitative trait loci in a Citrus grandis $\times$ Poncirus trifoliata $\mathrm{F}_{1}$ pseudo-testcross using molecular markers. J. Amer. Soc. Hort. Sci. 128:508-514.

Wisniewski, M., C. Bassett, and L.V. Gusta. 2003. An overview of cold hardiness in woody plants: Seeing the forest through the trees. HortScience 38:952-959.

Xin, Z. and J. Browse. 1998. eskimol mutants of Arabidopsis are constitutively freezing-tolerant. Proc. Nat. Acad. Sci. (USA) 95:7790-7804.

Zhang, J.Z., R.A. Creelman, and J.-K. Zhu. 2004. From laboratory to field. Using information from arabidopsis to engineer salt, cold, and drought tolerance in crops. Plant Physiol. 135:615-621. 